\title{
UPPER SEMICONTINUITY OF THE LAMINATION HULL
}

\author{
TERENCE L. J. HARRIS
}

\begin{abstract}
Let $K \subseteq \mathbb{R}^{2 \times 2}$ be a compact set, let $K^{r c}$ be its rank-one convex hull, and let $L(K)$ be its lamination convex hull. It is shown that the mapping $K \mapsto \overline{L(K)}$ is not upper semicontinuous on the diagonal matrices in $\mathbb{R}^{2 \times 2}$, which was a problem left by Kolár. This is followed by an example of a 5 -point set of $2 \times 2$ symmetric matrices with non-compact lamination hull. Finally, another 5 -point set $K$ is constructed, which has $L(K)$ connected, compact and strictly smaller than $K^{r c}$.
\end{abstract}

\section{INTRODUCTION}

Let $\mathbb{R}^{m \times n}$ denote the space of $m \times n$ matrices with real entries. Two matrices $X, Y \in \mathbb{R}^{m \times n}$ with $\operatorname{rank}(X-Y)=1$ are called rank-one connected. A set $\mathcal{S} \subseteq \mathbb{R}^{m \times n}$ is lamination convex if

$$
\lambda X+(1-\lambda) Y \in \mathcal{S} \text { for all } \lambda \in[0,1],
$$

whenever $X, Y \in \mathcal{S}$ are rank-one connected. For a set $K \subseteq \mathbb{R}^{m \times n}$, the smallest lamination convex set containing $K$ is denoted by $L(K)$.

This work contains a counterexample to a question posed in [5], concerning the continuity of the mapping $K \mapsto \overline{L(K)}$ on $\mathbb{R}^{2 \times 2}$. The example is similar to Example 2.2 in [1]. This is followed by a 5 -point set $K$ of symmetric $2 \times 2$ matrices with non-compact $L(K)$, similar to Example 2.4 in [5]. Then, another 5-point set $K$ is constructed which has $L(K)$ connected, compact and strictly smaller than $K^{r c}$. This is contrasted with Proposition 2.5 in [8], which says that $K^{p c}=L(K)=K$ if $K$ is connected, compact and has no rank-one connections. Finally, a weaker version of this result is given for sets with rank-one connections.

\section{MAin RESUlts}

Define the Hausdorff distance between two compact sets $K_{1}, K_{2}$ in $\mathbb{R}^{m \times n}$ by

$$
\rho\left(K_{1}, K_{2}\right)=\inf \left\{\epsilon \geq 0: K_{1} \subseteq U_{\epsilon}\left(K_{2}\right) \text { and } K_{2} \subseteq U_{\epsilon}\left(K_{1}\right)\right\},
$$

where $U_{\epsilon}(K)$ is the open $\epsilon$-neighbourhood of $K$, corresponding to the Euclidean distance. Let $\mathcal{K}$ be the set of compact subsets of $\mathbb{R}^{m \times n}$. A function $f: \mathcal{K} \rightarrow \mathcal{K}$ is upper semicontinuous if for every $\epsilon>0$ and for every $K_{0} \in \mathcal{K}$, there exists a $\delta>0$ such that $f(K) \subseteq U_{\epsilon}\left(f\left(K_{0}\right)\right)$ whenever $\rho\left(K, K_{0}\right)<\delta$. It is known that the function $K \mapsto K^{r c}$ is upper semicontinuous on the compact subsets of $\mathbb{R}^{m \times n}$ (see for example the proof of Theorem 1 in [7], Example 4.18 in [4], or Theorem 3.2 in

Key words and phrases. Lamination convexity, rank-one convexity.

Maths Subject Classification (2000): 49J45 and 52A30.

This research was supported by the Australian Research Council's Discovery Projects funding scheme (project DP140100531). 
9].) The following example (pictured in Figure 1) shows that this fails on diagonal matrices in $\mathbb{R}^{2 \times 2}$, for the lamination convex hull.

Theorem 2.1. There exists a compact set $K_{0}$ of diagonal matrices in $\mathbb{R}^{2 \times 2}$ such that the mapping $K \mapsto \overline{L(K)}$ is not upper semicontinuous at $K_{0}$.

Proof. Identify the space of $2 \times 2$ diagonal matrices with $\mathbb{R}^{2}$ in the natural way. Let

$$
K_{0}=\{(1,0)\} \cup \bigcup_{n=0}^{\infty}\left\{\left(1-\frac{3}{2^{n+1}}, \frac{1}{2^{n+1}}\right), \quad\left(1-\frac{1}{2^{n}}, \frac{3}{2^{n+1}}\right)\right\} .
$$

The set $K_{0}$ is compact and has no rank-one connections, thus $L\left(K_{0}\right)=K_{0}$. For each integer $n \geq-1$ let

$$
P_{n}=\left(1-\frac{1}{2^{n+1}}, \frac{1}{2^{n+1}}\right) \text {. }
$$

Given $\delta>0$, choose a positive integer $N$ large enough to ensure that $\frac{1}{2^{N}}<\delta$, and let $K=K_{0} \cup\left\{P_{N}\right\}$, so that $\rho\left(K, K_{0}\right)<\delta$. Then

$$
\left(1-\frac{1}{2^{N}}, \frac{1}{2^{N+1}}\right)=\frac{1}{2}\left(1-\frac{3}{2^{N+1}}, \frac{1}{2^{N+1}}\right)+\frac{1}{2} P_{N} \in L(K),
$$

and hence

$$
P_{N-1}=\frac{1}{2}\left(1-\frac{1}{2^{N}}, \frac{1}{2^{N+1}}\right)+\frac{1}{2}\left(1-\frac{1}{2^{N}}, \frac{3}{2^{N+1}}\right) \in L(K) .
$$

It follows by induction that $(0,1)=P_{-1} \in L(K)$. Since $\rho\left(P_{-1}, L\left(K_{0}\right)\right) \geq \frac{1}{2}$, this shows that the function $K \mapsto \overline{L(K)}$ is not upper semicontinuous at $K_{0}$.

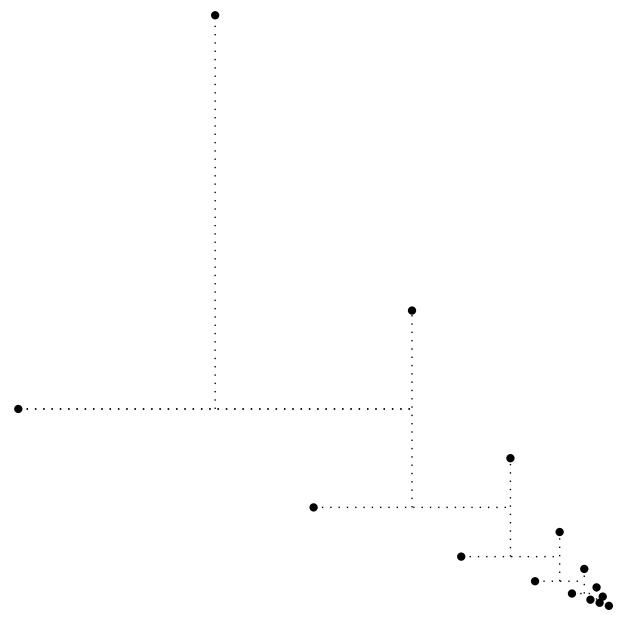

Figure 1. The set $K_{0}$ from Theorem 2.1. The dotted lines are rank-one lines in $L(K)$, where $K$ is a small perturbation of $K_{0}$.

The next result gives two examples of 5-point subsets of $\mathbb{R}^{2 \times 2}$, each with a noncompact lamination hull. The upper-triangular example is pictured in Figure 2, It consists of 4 points in the diagonal plane arranged in a $T_{4}$ configuration, together with a point whose projection onto the diagonal plane is a corner of the inner rectangle of the $T_{4}$ configuration. 
Throughout, the upper triangular matrix $\left(\begin{array}{ll}x & z \\ 0 & y\end{array}\right)$ will be identified with the point $(x, y, z) \in \mathbb{R}^{3}$. The symmetric example uses essentially the same idea as in Figure 2] so the matrix $\left(\begin{array}{ll}x & z \\ z & y\end{array}\right)$ will also be denoted by the point $(x, y, z) \in \mathbb{R}^{3}$. Since the cases are treated separately, the notations do not conflict. The symmetric notation also differs from the usual identification, used for example in [5]. The space of $2 \times 2$ upper triangular matrices is denoted by $\mathbb{R}_{\text {tri }}^{2 \times 2}$, and the space of $2 \times 2$ symmetric matrices by $\mathbb{R}_{\mathrm{sym}}^{2 \times 2}$. Up to linear isomorphisms preserving rank-one directions, these are the only two 3 -dimensional subspaces of $\mathbb{R}^{2 \times 2}$ (see 2, Corollary 6] or [6, Lemma 3.1])

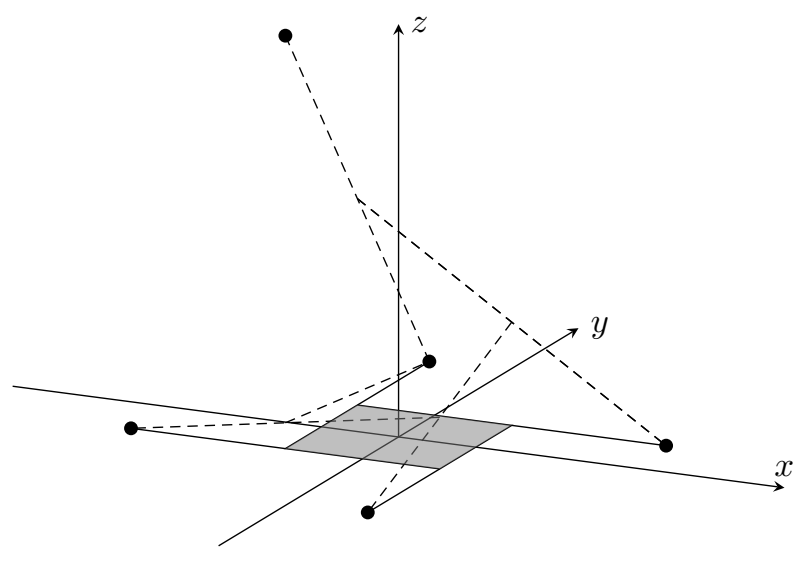

Figure 2. A 5 -point set $K \subseteq \mathbb{R}_{\text {tri }}^{2 \times 2}$ together with 5 rank-one lines in $L(K)$. The dashed lines indicate rank-one lines in $L(K)$, which spiral toward the diagonal plane and make $L(K)$ non-compact.

\section{Theorem 2.2.}

(i) There exists a 5-point set $K \subseteq \mathbb{R}_{\text {tri }}^{2 \times 2}$ such that $L(K)$ is not compact.

(ii) There exists a 5-point set $K \subseteq \mathbb{R}_{\text {sym }}^{2 \times 2}$ such that $L(K)$ is not compact.

Proof. For part (i) let $x_{1}<x_{2}, y_{2}<y_{1}, z_{0}>0$ and $\alpha_{0}, \alpha_{1}, \alpha_{2}, \alpha_{3}>0$. Let

$$
P_{0}=\left(x_{1}, y_{2}, 0\right), \quad P_{1}=\left(x_{1}, y_{1}, 0\right), \quad P_{2}=\left(x_{2}, y_{1}, 0\right), \quad P_{3}=\left(x_{2}, y_{2}, 0\right),
$$

and set

$$
\begin{array}{ll}
A_{0}=\left(x_{1}, y_{1}+\alpha_{0}, 0\right), & A_{1}=\left(x_{2}+\alpha_{1}, y_{1}, 0\right), \\
A_{2}=\left(x_{2}, y_{2}-\alpha_{2}, 0\right), & A_{3}=\left(x_{1}-\alpha_{3}, y_{2}, 0\right) .
\end{array}
$$

For $i \in\{0,1,2,3\}$ let $A_{4}=A_{0}$ and

$$
\lambda_{i}=\frac{\operatorname{det}\left(A_{i}-A_{i+1}\right)}{\operatorname{det}\left(A_{i}-A_{i+1}\right)-\operatorname{det}\left(P_{i}-A_{i+1}\right)} \in(0,1),
$$


let $X_{0}=P_{0}+\left(0,0, z_{0}\right)$ and $K=\left\{A_{0}, A_{1}, A_{2}, A_{3}, X_{0}\right\}$. For $i \geq 0$ let

$$
X_{i+1}=\left(1-\lambda_{i \bmod 4}\right) A_{i \bmod 4}+\lambda_{i \bmod 4} X_{i},
$$

so that for $i \geq 0$ and $k \in\{0,1,2,3\}$, induction gives

$$
X_{4 i+k}=P_{k}+\left(\lambda_{0} \lambda_{1} \lambda_{2} \lambda_{3}\right)^{i}\left(\prod_{j=0}^{k-1} \lambda_{j}\right)\left(0,0, z_{0}\right), \quad \operatorname{det}\left(X_{i}-A_{i \bmod 4}\right)=0,
$$

which implies that $X_{i} \in L(K)$ for every $i \geq 0$. Hence $P_{0} \in \overline{L(K)}$, and it remains to show that $P_{0} \notin L(K)$. This follows from the fact that

$$
\left\{(x, y, z) \in \mathbb{R}_{\mathrm{tri}}^{2 \times 2}: z>0\right\} \cup\left\{A_{0}, A_{1}, A_{2}, A_{3}\right\}
$$

is a lamination convex set containing $K$, which does not contain $P_{0}$.

For part (ii), let all the scalars and diagonal points be the same as in part (i). Using the symmetric notation let $Y_{0}=P_{0}+\left(\xi_{1}, \xi_{2}, \xi_{3}\right)$ where $\xi_{3}>0$ and

$$
\xi_{1}=\frac{1}{2}\left(-\alpha_{3}+\sqrt{\alpha_{3}^{2}-\frac{4 \alpha_{3} \xi_{3}^{2}}{y_{1}+\alpha_{0}-y_{2}}}\right), \quad \xi_{2}=\frac{-\xi_{1}\left(y_{1}+\alpha_{0}-y_{2}\right)}{\alpha_{3}} .
$$

so that $\operatorname{det}\left(Y_{0}-A_{0}\right)=\operatorname{det}\left(Y_{0}-A_{3}\right)=0$, and $Y_{0} \rightarrow P_{0}$ as $\xi_{3} \rightarrow 0$. The fact that $\operatorname{det}\left(P_{0}-A_{1}\right)>0>\operatorname{det}\left(A_{0}-A_{1}\right)$ means that

$$
\operatorname{det}\left(Y_{0}-A_{1}\right)>0>\operatorname{det}\left(A_{0}-A_{1}\right),
$$

whenever $\xi_{3} \in\left(0, \epsilon_{1}\right)$, for some $\epsilon_{1}>0$. Set $B_{0}=Y_{0}$. For $i \in\{0,1,2,3\}$ and $B_{i}$ with

$$
\operatorname{det}\left(B_{i}-A_{i+1}\right) \neq 0 \text { and } \operatorname{sgn} \operatorname{det}\left(B_{i}-A_{i+1}\right) \neq \operatorname{sgn} \operatorname{det}\left(A_{i}-A_{i+1}\right),
$$

let

$$
B_{i+1}=\left(1-t_{i}\right) A_{i}+t_{i} B_{i}, \text { where } t_{i}=\frac{\operatorname{det}\left(A_{i}-A_{i+1}\right)}{\operatorname{det}\left(A_{i}-A_{i+1}\right)-\operatorname{det}\left(B_{i}-A_{i+1}\right)} \in(0,1),
$$

so that $\operatorname{det}\left(B_{i+1}-A_{i+1}\right)=0$. By induction $t_{i} \rightarrow \lambda_{i}$ as $\xi_{3} \rightarrow 0$ for $i \in\{0,1,2,3\}$, $B_{i} \rightarrow P_{i \bmod 4}$ as $\xi_{3} \rightarrow 0$ for each $i \in\{0,1,2,3,4\}$, and $B_{1}, B_{2}, B_{3}, B_{4}$ all exist if $\xi_{3}$ is sufficiently small. Hence there exists $\epsilon_{2}>0$ such that $\left(t_{0} t_{1} t_{2} t_{3}\right)<\frac{1}{2}\left(1+\lambda_{0} \lambda_{1} \lambda_{2} \lambda_{3}\right)$ and $B_{1}, B_{2}, B_{3}, B_{4}$ all exist whenever $\xi_{3} \in\left(0, \epsilon_{2}\right)$. Put $\left(\eta_{1}, \eta_{2}, \eta_{3}\right)=B_{4}-P_{0}$. Then since $\operatorname{det}\left(B_{4}-A_{0}\right)=\operatorname{det}\left(B_{4}-A_{3}\right)=0$,

$$
\eta_{1}=\frac{1}{2}\left(-\alpha_{3} \pm \sqrt{\alpha_{3}^{2}-\frac{4 \alpha_{3} \eta_{3}^{2}}{y_{1}+\alpha_{0}-y_{2}}}\right), \quad \eta_{2}=\frac{-\eta_{1}\left(y_{1}+\alpha_{0}-y_{2}\right)}{\alpha_{3}} .
$$

But since $B_{4} \rightarrow P_{0}$ as $\xi_{3} \rightarrow 0$, there exists $\epsilon_{3}>0$ such that the sign in (2.1) is positive whenever $\xi_{3} \in\left(0, \epsilon_{3}\right)$. Let $\epsilon=\min \left\{\epsilon_{1}, \epsilon_{2}, \epsilon_{3}\right\}$. If $\xi_{3} \in(0, \epsilon)$. then

$$
\eta_{3}=\left(t_{0} t_{1} t_{2} t_{3}\right) \xi_{3}<\frac{1}{2}\left(1+\lambda_{0} \lambda_{1} \lambda_{2} \lambda_{3}\right) \xi_{3}
$$

Therefore let $K=\left\{A_{0}, A_{1}, A_{2}, A_{3}, Y_{0}\right\}$, and set $Y_{1}=B_{4}$. Then $Y_{1} \in L(K)$ by the preceding working. By (2.2), iterating this process gives a sequence $Y_{n} \in L(K)$ with $Y_{n} \rightarrow P_{0} \in \overline{L(K)}$. Again the point $P_{0}$ is not in $L(K)$ since

$$
\left\{(x, y, z) \in \mathbb{R}_{\mathrm{sym}}^{2 \times 2}: z>0\right\} \cup\left\{A_{0}, A_{1}, A_{2}, A_{3}\right\}
$$

is a lamination convex set separating $P_{0}$ from $K$. Hence $L(K)$ is not compact. 
A function $f: \mathbb{R}^{m \times n} \rightarrow \mathbb{R}$ is called rank-one convex if

$$
f(\lambda X+(1-\lambda) Y) \leq \lambda f(X)+(1-\lambda) f(Y) \text { for all } \lambda \in[0,1],
$$

whenever $\operatorname{rank}(X-Y) \leq 1$. The rank-one convex hull of a compact set $K \subseteq \mathbb{R}^{m \times n}$ is defined by

$$
K^{r c}=\left\{X \in \mathbb{R}^{m \times n}: f(X) \leq 0 \quad \forall \text { rank-one convex } f \text { with }\left.f\right|_{K} \leq 0\right\} .
$$

The polyconvex hull is defined similarly via polyconvex functions; a function $f: \mathbb{R}^{2 \times 2} \rightarrow \mathbb{R}$ is polyconvex if there exists a convex function $g: \mathbb{R}^{2 \times 2} \times \mathbb{R} \rightarrow \mathbb{R}$ such that $f(X)=g(X$, $\operatorname{det} X)$ for all $X \in \mathbb{R}^{2 \times 2}$. For compact $K$, the following characterisation of $K^{p c}$ will be used (see Theorem 1.9 in [4]):

$$
K^{p c}=\left\{\bar{\mu}: \mu \in \mathscr{M}_{p c}(K)\right\},
$$

where $\mathscr{M}_{p c}(K)$ is the class of probability measures supported in $K$ which satisfy Jensen's inequality for all polyconvex $f$;

$$
f(\bar{\mu}) \leq \int_{\mathbb{R}^{2 \times 2}} f(X) d \mu(X) \quad \text { where } \quad \bar{\mu}=\int_{\mathbb{R}^{2 \times 2}} X d \mu(X) .
$$

Definition 2.3. An ordered set $\left\{X_{i}\right\}_{i=1}^{4} \subseteq \mathbb{R}^{m \times n}$ without rank-one connections is called a $T_{4}$ configuration if there exist matrices $P, C_{1}, C_{2}, C_{3}, C_{4} \in \mathbb{R}^{m \times n}$ and real numbers $\mu_{1}, \mu_{2}, \mu_{3}, \mu_{4}>1$ satisfying

$$
\operatorname{rank} C_{i}=1 \text { for } 1 \leq i \leq 4, \quad \sum_{i=1}^{4} C_{i}=0,
$$

and

$$
\begin{aligned}
& X_{1}=P+\mu_{1} C_{1} \\
& X_{2}=P+C_{1}+\mu_{2} C_{2} \\
& X_{3}=P+C_{1}+C_{2}+\mu_{3} C_{3} \\
& X_{4}=P+C_{1}+C_{2}+C_{3}+\mu_{4} C_{4} .
\end{aligned}
$$

An unordered set $\left\{X_{i}\right\}_{i=1}^{4}$ is a $T_{4}$ configuration if it has at least one ordering which is a $T_{4}$ configuration.

The following result is a slight generalisation of Theorem 1 in [7] (see also Corollary 3 in [3] ). The proof is similar to the one in [7], with minor technical changes.

Theorem 2.4. If $K \subseteq \mathbb{R}^{2 \times 2}$ is compact, and does not have a $T_{4}$ configuration $\left\{X_{i}\right\}_{i=1}^{4}$ with at least two $X_{i}, X_{j}$ in distinct connected components of $L(K)$, then

$$
K^{r c}=\bigcup_{i}\left(U_{i} \cap K\right)^{r c} \quad \text { and } \quad K^{q c}=\bigcup_{i}\left(U_{i} \cap K\right)^{q c},
$$

where the $U_{i}$ are the connected components of $L(K)$.

On diagonal matrices the conclusion reduces to $K^{r c}=L(K)$. The following proposition shows that this fails in the full space $\mathbb{R}^{2 \times 2}$.

Proposition 2.5. There exists a 5-point set $K \subseteq \mathbb{R}^{2 \times 2}$ with $L(K)$ connected, compact and strictly smaller than $K^{\text {rc }}$. 
Proof. Fix $\epsilon \in(0,1)$, let

$$
X_{1}=\left(\begin{array}{ll}
1 & 0 \\
0 & 0
\end{array}\right), \quad X_{2}=\left(\begin{array}{ll}
0 & 0 \\
0 & 1
\end{array}\right), \quad X_{3}=\left(\begin{array}{cc}
-\epsilon & -1 \\
-\epsilon^{2} & -\epsilon
\end{array}\right), \quad X_{4}=\left(\begin{array}{cc}
-\epsilon & \epsilon^{2} \\
1 & -\epsilon
\end{array}\right),
$$

and let

$$
\mu_{1}=\frac{1+2 \epsilon}{\epsilon\left(1-\epsilon^{2}\right)}, \quad \mu_{2}=1+\epsilon^{2} \mu_{1}, \quad \mu_{3}=1+\left(\frac{1+\epsilon^{2}}{\epsilon}\right) \mu_{2}, \quad \mu_{4}=1+\epsilon^{2} \mu_{3},
$$

so that

$$
\mu_{1}=1+\frac{\mu_{4}}{\epsilon\left(1+\epsilon^{2}\right)}
$$

Set

$$
\begin{aligned}
& P_{1}=\frac{1}{\epsilon\left(\mu_{1}-1\right)}\left(\begin{array}{cc}
-\epsilon & 0 \\
1 & 0
\end{array}\right), \quad P_{2}=\frac{1}{\mu_{1} \epsilon}\left(\begin{array}{ll}
0 & 0 \\
1 & 0
\end{array}\right), \\
& P_{3}=\frac{1}{\mu_{2}}\left(\begin{array}{ll}
0 & 0 \\
\epsilon & 1
\end{array}\right), \quad P_{4}=\frac{1}{\mu_{3} \epsilon}\left(\begin{array}{cc}
-\epsilon^{2} & -\epsilon \\
\epsilon & 1
\end{array}\right),
\end{aligned}
$$

and let $C_{i}=P_{i+1}-P_{i}$, where $P_{5}:=P_{1}$. Then clearly $\operatorname{rank} C_{i}=1$ for all $i$, whilst (2.5) and (2.6) imply that this is a solution of (2.4). Let

$$
K=\left\{0, X_{1}, X_{2}, X_{3}, X_{4}\right\}, \quad \text { so that } \quad L(K)=\bigcup_{i=1}^{4}\left[0, X_{i}\right] .
$$

To prove the second formula for $L(K)$, it suffices to show that the set $\mathcal{S}=\bigcup_{i=1}^{4}\left[0, X_{i}\right]$ is lamination convex. For $i \neq j$, the fact that $\operatorname{det} X_{i}=\operatorname{det} X_{j}=0$ and $\operatorname{det}\left(X_{i}-X_{j}\right) \neq 0$ implies that $\operatorname{det}\left(X_{i}-t X_{j}\right) \neq 0$ whenever $t \in(0,1]$, since the determinant is linear along rank-one lines. It follows similarly that $\operatorname{det}\left(s X_{i}-t X_{j}\right) \neq 0$ for $s, t \in(0,1]$, and so the only rank-one connected pairs in $\mathcal{S}$ are 0 and $t X_{i}$ for any $i$. Hence $\mathcal{S}$ is lamination convex. By Lemma 2 in [7], the point $P_{1}$ is in $K^{r c} \backslash L(K)$, so this proves the proposition.

The preceding example contrasts with Lemma 3 in [8], which states (in a weakened form) that $K^{p c}=K$ if $K$ is a connected compact subset of $\mathbb{R}^{2 \times 2}$ without rankone connections. The example shows that the assumption that $K$ has no rank-one connections cannot be weakened to $L(K)=K$. The reason is that $\operatorname{det}(X-Y)$ cannot change sign on connected subsets of $\mathbb{R}^{2 \times 2}$ without rank-one connections, whilst it can on lamination convex sets. If the assumption that $\operatorname{det}(X-Y)$ does not change sign is added, $K^{p c}$ is equal to the lamination hull of order 2: given a set $K \subseteq \mathbb{R}^{m \times n}$, let $L^{(0)}(K)=K$ and define $L^{(k)}(K)$ inductively by

$$
L^{(k+1)}(K)=\bigcup_{\substack{X, Y \in L^{(k)}(K) \\ \operatorname{rank}(X-Y) \leq 1}}[X, Y] .
$$

Proposition 2.6. If $K \subseteq \mathbb{R}^{2 \times 2}$ is a compact set such that $\operatorname{det}(X-Y) \geq 0$ for every $X, Y \in K$, then $K^{p c}=L^{(2)}(K)$.

Proof. If $\mu$ is a probability measure supported in $K$ with $\operatorname{det} \bar{\mu}=\int_{\mathbb{R}^{2 \times 2}} \operatorname{det} X d \mu$, then as in [8],

$$
\int_{\mathbb{R}^{2 \times 2}} \int_{\mathbb{R}^{2 \times 2}} \operatorname{det}(X-Y) d \mu(X) d \mu(Y)=0
$$


and therefore $\operatorname{det}(X-Y)=0$ whenever $X$ and $Y$ are in the support of $\mu$. This implies (see the following Lemma 2.7) that the support of $\mu$ is contained in a 2dimensional affine plane $P$ consisting only of rank-one directions. Therefore $\bar{\mu} \in$ $(K \cap P)^{c o}$, and so Carathéodory's Theorem gives 3 points $X_{i} \in K \cap P$ such that $\bar{\mu}$ is a convex combination $\bar{\mu}=\lambda_{1} X_{1}+\lambda_{2} X_{2}+\lambda_{3} X_{3}$, and without loss of generality $\lambda_{1} \neq 0$. Then $\frac{\lambda_{1}}{\lambda_{1}+\lambda_{2}} \cdot X_{1}+\frac{\lambda_{2}}{\lambda_{1}+\lambda_{2}} \cdot X_{2} \in P \cap L^{(1)}(K)$ since $P$ is a plane consisting of rank-one directions, and similarly

$$
\bar{\mu}=\left(\lambda_{1}+\lambda_{2}\right)\left(\frac{\lambda_{1}}{\lambda_{1}+\lambda_{2}} \cdot X_{1}+\frac{\lambda_{2}}{\lambda_{1}+\lambda_{2}} \cdot X_{2}\right)+\lambda_{3} X_{3} \in L^{(2)}(K) .
$$

It follows from (2.3) that $K^{p c}=L^{(2)}(K)$.

Lemma 2.7. Let $X_{0}, Y_{0} \in \mathbb{R}^{m \times n}$ satisfy $\operatorname{rank}\left(X_{0}-Y_{0}\right)=1$, and let

$$
\mathcal{S}=\left\{X \in \mathbb{R}^{m \times n}: \operatorname{rank}\left(X-X_{0}\right) \leq 1 \text { and } \operatorname{rank}\left(X-Y_{0}\right) \leq 1\right\} .
$$

Then:

(i) $\mathcal{S}=P_{1} \cup P_{2}$, where $P_{1}$ is an $m$-dimensional affine plane and $P_{2}$ is an $n$ dimensional affine plane, and for each fixed $i, \operatorname{rank}(X-Y) \leq 1$ for $X, Y \in P_{i}$.

(ii) The planes $P_{1}$ and $P_{2}$ satisfy

$$
\operatorname{rank}(X-Y)>1 \quad \text { for } \quad X \in P_{1} \backslash P_{2} \quad \text { and } \quad Y \in P_{2} \backslash P_{1} \text {. }
$$

Proof. By translation invariance it may be assumed that $Y_{0}=0$, so that $\operatorname{rank} X_{0}=1$ and $X_{0}=v_{0} w_{0}^{T}$ for some nonzero $v_{0} \in \mathbb{R}^{m}, w_{0} \in \mathbb{R}^{n}$. Let

$$
P_{1}=\left\{x w_{0}^{T}: x \in \mathbb{R}^{m}\right\}, \quad P_{2}=\left\{v_{0} y^{T}: y \in \mathbb{R}^{n}\right\} .
$$

If $X \in \mathcal{S}$ then $X=v w^{T}$ for some $v \in \mathbb{R}^{m}$ and $w \in \mathbb{R}^{n}$, and

$$
X-X_{0}=v w^{T}-v_{0} w_{0}^{T}=a b^{T},
$$

for some $a \in \mathbb{R}^{m}$ and $b \in \mathbb{R}^{n}$. Suppose for a contradiction that $X \notin P_{1} \cup P_{2}$. Then since $X \notin P_{1}$ there exists a vector $w_{0}^{\perp}$ such that $\left\langle w_{0}, w_{0}^{\perp}\right\rangle=0$ and $\left\langle w, w_{0}^{\perp}\right\rangle \neq 0$. Right multiplying both sides of (2.7) with $w_{0}^{\perp}$ gives

$$
v=\frac{\left\langle b, w_{0}^{\perp}\right\rangle a}{\left\langle w, w_{0}^{\perp}\right\rangle}, \quad \text { and similarly } \quad w=\frac{\left\langle a, v_{0}^{\perp}\right\rangle b}{\left\langle v, v_{0}^{\perp}\right\rangle} .
$$

Let $\lambda=\frac{\left\langle a, v_{0}^{\perp}\right\rangle\left\langle b, w_{0}^{\perp}\right\rangle}{\left\langle v, v_{0}^{\perp}\right\rangle\left\langle w, w_{0}^{\perp}\right\rangle}$. Then $\lambda \neq 1$ by (2.7) since $v_{0} w_{0}^{T} \neq 0$, and therefore

$$
X=v w^{T}=\left(\frac{\lambda}{\lambda-1}\right) v_{0} w_{0}^{T} \in P_{1} \cap P_{2}
$$

which is a contradiction. This proves part (i).

For part (ii), let $X=x w_{0}^{T} \in P_{1} \backslash P_{2}$, let $Y=v_{0} y^{T} \in P_{2} \backslash P_{1}$ and suppose for a contradiction that $\operatorname{rank}(X-Y)=1$. Then by part (i), $Y=x z^{T}$ for some nonzero $z \in \mathbb{R}^{n}$, and therefore $x=\frac{v_{0}\langle y, z\rangle}{\|z\|^{2}}$, which contradicts the fact that $X \notin P_{2}$.

\section{ACKNOWLEDGEMENTS}

I would like to thank Michael Cowling and Alessandro Ottazzi for advice on this topic, and for comments on the draft. 


\section{REFERENCES}

[1] Aumann, R.J., Hart, S.: Bi-convexity and bi-martingales. Israel J. Math. 54, 159-180 (1986)

[2] Conti, S., Faraco, D., Maggi, F., Müller, S.: Rank-one convex functions on $2 \times 2$ symmetric matrices and laminates on rank-three lines. Calc. Var. Partial Differential Equations 24, 479-493 (2005)

[3] Faraco, D., Székelyhidi Jr, L.: Tartar's conjecture and localization of the quasiconvex hull in $\mathbb{R}^{2 \times 2}$. Acta Math. 200(2), 279-305 (2008)

[4] Kirchheim, B.: Rigidity and geometry of microstructures. Habilitation thesis, University of Leipzig (2003)

[5] Kolář, J.: Non-compact lamination convex hulls. Ann. Inst. H. Poincaré Anal. Non Linéaire 20(3), 391-403 (2003)

[6] Kreiner, C.F., Zimmer, J.: Topology and geometry of nontrivial rank-one convex hulls for two-by-two matrices. ESAIM Control Optim. Calc. Var. 12, 253-270 (2006)

[7] Székelyhidi Jr, L.: Rank-one convex hulls in $\mathbb{R}^{2 \times 2}$. Calc. Var. Partial Differential Equations 22, 253-281 (2005)

[8] Šverák, V.: On Tartar's conjecture. Ann. Inst. H. Poincaré Anal. Non Linéaire 10, 405-412 (1993)

[9] Zhang, K.: On the stability of quasiconvex hulls. Preprint, Max-Plank Inst. for Mathematics in the Sciences, Leipzig, 33 (1998)

School of Mathematics and Statistics, University of New South Wales, Sydney, NSW 2052, Australia.

Department of Mathematics, University of Illinois, Urbana, IL 61801, U.S.A.

E-mail address: terence2@illinois.edu 